\title{
On a fixed point theorem for the product of operators
}

\author{
Mieczysław Cichoń and Mohamed M. A. Metwali
}

\begin{abstract}
In this paper, we study the existence of fixed points for the product of nonlinear operators. This kind of fixed point theorems is necessary in consideration of quadratic differential and integral problems. We emphasize a possible extension of the applicability of obtained theorems and consequently we prove the existence of fixed points for operators acting on some function spaces that are not necessarily Banach algebras. This result can be successfully applied to many quadratic problems.
\end{abstract}

Mathematics Subject Classification. 46E30, 45G10, 47H30, 47N20.

Keywords. Fixed point theorem, function spaces, pointwise multiplication, quadratic integral equations.

\section{Introduction}

In this paper we discuss a fixed point theorem for the product (pointwise multiplication) of two, possibly nonlinear, operators $A \cdot B$, i.e., for the problem

$$
x=g+\lambda \cdot A(x) \cdot B(x) .
$$

In contrast to the case of the sum of two operators $A+B$ (called the Krasnoselskii-type fixed point theorem), the proposed case is not sufficiently investigated. However, such a kind of theorems is also discussed and they are related to some "quadratic" problems. Let us mention the quadratic integral equation

$$
x(t)=g(t)+\lambda \cdot u(t, x(t)) \cdot \int_{0}^{1} K(t, s) f(s, x(s)) d s
$$

considered in $[13,14,17,18]$, for instance.

Let us stress one aspect of the existing results: all of them are related to operators acting on Banach algebras (with respect to the pointwise multiplication). It is a significant limitation, but unfortunately it is assumed in almost all earlier results of this type. In particular, it implies that for the 
above problems only continuous, $B V$ or even classical $C^{1}$, solutions can be found. Moreover, this approach leads to some additional restrictive assumption on the growth of considered operators. This is particularly clear when we put $A$ (or $B$ ) as the Nemytskii superposition operator, for which acting conditions are strictly related to the growth assumptions (cf. [18]). In our approach we indicate how to avoid this problem. We investigate two cases. The first one is when operators are contractions with respect to some measures of noncompactness. In the second case at least one of the operators should be a contraction with respect to the measure of uniform integrability $c$, which is a less restrictive condition. Some connections between the two cases will be discussed, by illustrating the advantage of our approach. Finally, as an example of applications, we prove the existence of positive monotone solutions for some quadratic integral equations.

\section{Pointwise multiplication in function spaces}

Let $\mathbb{R}$ be the field of real numbers. In this paper we denote by $I$ a compact interval $[0,1] \subset \mathbb{R}$. If we consider the pointwise product of two functions from a given function space $E$, then either the result is still in the same space (a Banach algebra case) or the space $E$ has no such a nice property. Let us observe that in the first case, for the product of two operators $A$ and $B$, the assumption that they are acting on the same space $E$ as well as their images are in $E$ is very restrictive. In particular, it implies some restrictions on the growth condition for both operators. A typical situation considered in many problems is when $A$ is the Nemytskii superposition operator. It continuously maps $L^{p}(I)$ into $L^{q}(I)$ if and only if

$$
|f(t, x)| \leq a(t)+b \cdot|x|^{p / q}
$$

for all $t \in I$ and $x \in \mathbb{R}$, where $a \in L_{q}(I)$ and $b \geq 0$. This is much simpler than the assumption that $A: C(I) \rightarrow C(I)$ is continuous.

In this paper some properties of the considered function spaces play a major role. We need to consider the triples of spaces with the following property: for a triple of spaces $\left(E, E_{1}, E_{2}\right)$ there exists a constant $k$ such that for arbitrary $x \in E_{1}$ and $y \in E_{2}$,

$$
x \cdot y \in E
$$

and

$$
\|x \cdot y\|_{E} \leq k \cdot\|x\|_{E_{1}} \cdot\|y\|_{E_{2}} .
$$

Let us recall some special cases. Most known is the case of Banach algebras, i.e., the space of continuous functions. In this case $E=E_{1}=E_{2}=C(I, X)$ $(k=1$, or so-called quasi-algebras for $k \neq 1)$. Moreover, some subalgebras of this space can be interesting, but we are not restricted only to this case. Let us note that the space of real-valued functions of finite total variation is a Banach (quasi-) algebra with $k=2$ (see [16]).

However, if we still need to consider "bigger" spaces, we need to go outside the class of Banach (quasi-) algebras and our extension will be useful. 
For discontinuous functions, let us recall Hölder's inequality for Lebesgue spaces:

$$
\|x \cdot y\|_{L^{1}} \leq\|x\|_{L^{p}} \cdot\|y\|_{L^{q}}
$$

whenever $1 / p+1 / q=1$. Thus, the triple $\left(L^{1}, L^{p}, L^{q}\right)$ is good enough.

The third example (and most important) is devoted to study some Orlicz spaces (for necessary definitions see [28, 30], for instance). Generally speaking, the product of two functions $x, y \in L_{M}(I)$ is not in $L_{M}(I)$. However, if $x$ and $y$ belong to some particular Orlicz spaces, then the product $x \cdot y$ belongs to a third Orlicz space. Let us note that one can find two functions belonging to Orlicz spaces: $u \in L_{U}(I)$ and $v \in L_{V}(I)$ such that the product $u v$ does not belong to any Orlicz space (this product is not integrable). Nevertheless, we have the following lemma.

Lemma 2.1 (See [28, Lemma 13.5], [30, Theorem 10.2]). Let $\varphi_{1}, \varphi_{2}$ and $\varphi$ be arbitrary $N$-functions. The following conditions are equivalent:

(1) for every functions $u \in L_{\varphi_{1}}(I)$ and $w \in L_{\varphi_{2}}(I), u \cdot w \in L_{\varphi}(I)$;

(2) there exists a constant $k>0$ such that for all measurable $u, w$ on $I$ we have $\|u w\|_{\varphi} \leq k\|u\|_{\varphi_{1}}\|w\|_{\varphi_{2}}$

(3) there exist numbers $C>0, u_{0} \geq 0$ such that for all $s, t \geq u_{0}$,

$$
\varphi\left(\frac{s t}{C}\right) \leq \varphi_{1}(s)+\varphi_{2}(t) ;
$$

(4) the functions $\varphi, \varphi_{1}$ and $\varphi_{2}$ satisfy the condition

$$
\limsup _{t \rightarrow \infty} \frac{\varphi_{1}^{-1}(t) \varphi_{2}^{-1}(t)}{\varphi(t)}<\infty .
$$

Let us recall the following simple sufficient condition for statements (1)-(4) to hold true.

Lemma 2.2 (See $\left[28\right.$, p. 223]). If there exist complementary $N$-functions $Q_{1}$ and $Q_{2}$ such that the inequalities

$$
\begin{aligned}
Q_{1}(\alpha u) & <\varphi^{-1}\left[\varphi_{1}(u)\right], \\
Q_{2}(\alpha u) & <\varphi^{-1}\left[\varphi_{2}(u)\right]
\end{aligned}
$$

hold, then for every functions $u \in L_{\varphi_{1}}(I)$ and $w \in L_{\varphi_{2}}, u \cdot w \in L_{\varphi}(I)$. If, moreover, $\varphi$ satisfies the $\Delta_{2}$-condition, then it is sufficient that the inequalities

$$
\begin{aligned}
& Q_{1}(\alpha u)<\varphi_{1}\left[\varphi^{-1}(u)\right], \\
& Q_{2}(\alpha u)<\varphi_{2}\left[\varphi^{-1}(u)\right]
\end{aligned}
$$

hold.

An interesting discussion about necessary and sufficient conditions for product operators can be found in [28, Section 13] and [30]. Note that, since $L^{p}=L_{M}$ for $M(t)=t^{p} / p$, the case of Lebesgue spaces $L^{p}$ is included in the above consideration for Orlicz spaces.

We will use a general concept of function spaces, i.e., ideal spaces (or Köthe function spaces). A normed space $(X,\|\cdot\|)$ of (classes of) measurable 
functions $x: I \rightarrow U$ ( $U$ is a normed space) is called preideal if for each $x \in X$ and each measurable $y: I \rightarrow U$, the relation $|y(s)| \leq|x(s)|$ (for almost all $s \in I)$ implies $y \in X$ and $\|y\| \leq\|x\|$. If $X$ is also complete, it is called an ideal space (see [32]). An ideal normed space $X$ is called regular if all singletons in $X$ have equicontinuous norm, i.e.,

$$
\lim _{\delta \rightarrow 0} \sup _{\{D: \text { meas } D \leq \delta\}}\left\|x \cdot \chi_{D}\right\|=0,
$$

where $\chi_{D}$ is the characteristic function of a measurable set $D$ (cf. $[22,33,34]$ ). Examples of regular ideal spaces: the Lebesgue spaces, the Orlicz spaces or the Lorentz spaces (with suitable norms). Note that the space of continuous functions $C(I)$ is not ideal, although it is a closed subspace of an ideal space.

We say that a set $T$ in an ideal space $E$ is compact in measure if it is compact in the topology of convergence in measure, i.e., as a subset of the space of all measurable functions $L^{0}(I)$ (see [25, 32]).

Finally, we can consider also a special case for $E_{2}=L^{\infty}$ and some function spaces for which $\left(E=E_{1}\right)$

$$
\|x \cdot y\|_{E} \leq\|x\|_{E} \cdot\|y\|_{L^{\infty}} .
$$

The class of spaces with this property is known as preideal* spaces (cf. [34, p. 66] or [32]). Although this case seems to be general, it has, from our point of view, one weakness: the measure of noncompactness in $L^{\infty}$ allowing us to prove our version of the fixed point theorem seems to be inapplicable and we do not discuss it in this paper. Moreover, any continuous superposition operator with values in $L^{\infty}$ should be constant. In particular, the function $u$ in equation (1.1) should be not depending on $x$, so the problem is reduced to standard (not quadratic) integral equations.

In our considerations, we will not be restricted to the cases presented above, but they confirm our belief that this kind of assumptions is really natural. It is possible to check this property for a given triple of function spaces. An open question is if it is possible to characterize all such spaces.

\section{Measures of noncompactness}

If $X$ is a subset of a Banach space $E$, then $\bar{X}$ and conv $X$ denote the closure and convex closure of $X$, respectively. By $B_{r}$ we denote a ball centered at $\theta$ with radius $r$. The standard algebraic operations on sets will be denoted by the symbols $k \cdot X$ and $X+Y$.

Moreover, by $\mathcal{M}_{E}$ we denote the family of all nonempty and bounded subsets of $E$ and by $\mathcal{N}_{E}$ its subfamily consisting of all relatively compact subsets. We will apply an axiomatic approach to the notion of a measure of noncompactness.

Definition 3.1 (See [11]). A mapping $\mu: \mathcal{M}_{E} \rightarrow[0, \infty)$ is said to be a measure of noncompactness in $E$ if it satisfies the following conditions:

(i) $\mu(X)=0 \Rightarrow X \in \mathcal{N}_{E}$;

(ii) $X \subset Y \Rightarrow \mu(X) \leq \mu(Y)$; 
(iii) $\mu(\bar{X})=\mu(\operatorname{conv} X)=\mu(X)$;

(iv) $\mu(\lambda X)=|\lambda| \mu(X)$ for $\lambda \in \mathbb{R}$;

(v) $\mu(X+Y) \leq \mu(X)+\mu(Y)$;

(vi) $\mu(X \cup Y)=\max \{\mu(X), \mu(Y)\}$;

(vii) if $X_{n}$ is a sequence of nonempty, bounded, closed subsets of $E$ such that $X_{n+1} \subset X_{n}, n=1,2,3, \ldots$, and $\lim _{n \rightarrow \infty} \mu\left(X_{n}\right)=0$, then the set $X_{\infty}=\bigcap_{n=1}^{\infty} X_{n}$ is nonempty.

We will call the family of sets $A$ with $\mu(A)=0$ a kernel of the measure and we will denote it by ker $\mu$. Let us recall a classical example: the Hausdorff measure of noncompactness $\beta_{H}(X)$ (cf. [11]) is defined as follows:

$$
\begin{array}{r}
\beta_{H}(X)=\inf \{r>0: \text { there exists a finite subset } Y \\
\text { of } \left.E \text { such that } X \subset Y+B_{r}\right\},
\end{array}
$$

where $X$ is an arbitrary nonempty and bounded subset of $E$. For more examples, we refer the reader to [11].

To distinguish between some measures of noncompactness $\mu$ in different spaces (if necessary) we will indicate an appropriate space as an index, i.e., $\mu_{E}, \mu_{E_{1}}$, etc.

Let $c$ denote the measure of uniform integrability of the set $X$ in an ideal function space $E$ on the compact interval $I$ (introduced in [5], cf. also [34, Definition 3.9] or $[22,23])$ :

$$
c(X)=\limsup _{\varepsilon \rightarrow 0} \sup _{\operatorname{mes} D \leq \varepsilon} \sup _{x \in X}\left\|x \cdot \chi_{D}\right\|_{E},
$$

where $\chi_{D}$ denotes the characteristic function of a measurable subset $D \subset I$.

The following proposition clarify the connections between the two coefficients in regular ideal spaces.

Proposition 3.2 (See [23, Theorem 1]). Let $X$ be a nonempty, bounded and compact in measure subset of an ideal regular space $E$. Then

$$
\beta_{H}(X)=c(X) .
$$

It is worth noting that in the context of Orlicz spaces the above proposition follows from [28, Theorem 11.3]. As a consequence, we obtain that bounded sets which are additionally compact in measure are compact in $E$ if and only if they are equi-integrable in this space, i.e., they have equiabsolutely continuous norms, in particular when $X$ is a subset of a regular part of $E$. This useful property will help us to replace weak (sequential) continuity conditions for considered operators by a strong one, which seems to be also interesting in many cases, but let us concentrate here on the strong topology.

\section{Main results}

Since we are interested in the existence of fixed points of some product operators, we will assume that our operators have values in some intermediate 
spaces $E_{1}$ and $E_{2}$ and then the product will again turn the values into the original space $E$ for the triple $\left(E, E_{1}, E_{2}\right)$, as in (2.2).

First, let us apply our approach to the most applicable theorem of this type. Consider an arbitrary (in the sense of Definition 3.1) measure of noncompactness $\mu$ in $C(I, E)$. An interesting fixed point theorem for operators acting on Banach algebras was proved by Banaś and Lecko in [12].

Let us consider different spaces of functions with a suitable choice of measures of noncompactness $\mu_{E}$ on $E, \mu_{E_{1}}$ on $E_{1}$ and $\mu_{E_{2}}$ on $E_{2}$ satisfying the axioms in Definition 3.1. We illustrate our approach by presenting an immediate extension of earlier results, i.e., for spaces that are not necessarily Banach algebras.

Theorem 4.1. Let $E, E_{1}, E_{2}$ be Banach spaces. Assume that $T \subset B_{r}(E)$ is a nonempty, bounded, closed and convex subset of the Banach space $E$, and that $g: B_{r}(E) \rightarrow B_{r}(E)$ has an absolutely continuous norm in E. Moreover, assume that $A: E \rightarrow E_{1}$ and $B: E \rightarrow E_{2}$ and that

(A1) $A$ continuously transforms the set $T$ into $T_{1} \subset E_{1}$ and $A\left(B_{r}(E)\right)$ is bounded in $E_{1}$,

(A2) there exists a constant $k_{1}>0$ such that $A$ satisfies the inequality

$$
\mu_{E_{1}}(A(U)) \leq k_{1} \cdot \mu_{E}(U)
$$

for arbitrary bounded subset $U$ of $E$,

(B1) $B$ continuously transforms the set $T$ into $T_{2} \subset E_{2}$ and $B\left(B_{r}(E)\right)$ is bounded in $E_{2}$,

(B2) there exists a constant $k_{2}>0$ such that $B$ satisfies the inequality

$$
\mu_{E_{2}}(B(U)) \leq k_{2} \cdot \mu_{E}(U)
$$

for arbitrary bounded subset $U$ of $E$,

(E1) for the triple of spaces $\left(E, E_{1}, E_{2}\right)$ there exists a constant $k$ such that for arbitrary $x \in E_{1}, y \in E_{2}$ and $t \in I$,

$$
x \cdot y \in E
$$

and

$$
\|x \cdot y\|_{E} \leq k \cdot\|x\|_{E_{1}} \cdot\|y\|_{E_{2}},
$$

(E2) $\lambda$ and $g$ are such that

$$
\|g\|_{E}+\lambda \cdot k \cdot \sup _{x \in B_{r}(E)}\|A(x)\|_{E_{1}} \cdot \sup _{x \in B_{r}(E)}\|A(x)\|_{E_{2}} \leq r
$$

for any $x \in T$ and $g+\lambda A(x) \cdot B(x) \in T$,

(C) $\sup _{x \in T}\|A(x)\|_{E_{1}} \cdot k_{2} \cdot|\lambda|+\sup _{x \in T}\|B(x)\|_{E_{2}} \cdot k_{1} \cdot|\lambda|<1$.

Then there exists at least one fixed point for the operator $K=g+\lambda \cdot A \cdot B$ in the set $T$ and the set of all fixed points of $K$ belongs to the kernel ker $\mu_{E}$.

This theorem was proved by Banaś in a special case of Banach algebras $E=E_{1}=E_{2}=C(I)=C(I, \mathbb{R})(k=1)$ (cf. also [20, 21], for instance). We do not require that the values of all operators are from the same space, but by using property $(2.2)$ of the considered spaces we are able to repeat the 
proof, so let us omit the details. However, a detailed proof will be presented for the next theorem under more general assumptions.

Note that if the above result is used for proving the existence of continuous solutions (i.e., $E=C(I, \mathbb{R})$ ), then the Ascoli criterion of compactness simplifies the proof, because the norm convergence of sequences is directly related with their pointwise convergence. Our approach allows to use different criteria of compactness (or even weak compactness!) in different ideal spaces (cf. $[18,17])$.

Now, we will consider the case of functions spaces without such a nice property. Namely, some subspaces of a space $L^{0}(I)$ of measurable functions bigger than $C(I)$ will be investigated. It allows us to apply our fixed point theorem to the problems with discontinuous solutions. This proof will be based on different compactness criteria than the earlier one (the Dunford-Pettis theorem and the Erzakova theorem). We do not assume that the considered operators are contractions with respect to the measure of noncompactness in $E$ (however one of them should be a contraction with respect to the measure $c$, see a remark after the proof), but their domain will be assumed to be additionally compact in measure (cf. [25, 27]).

Theorem 4.2. Assume that $T \subset B_{r}(E)$ is a nonempty, bounded, closed, convex and compact in measure subset of a regular ideal function space $E$, $g: B_{r}(E) \rightarrow B_{r}(E)$ has an absolutely continuous norm in $E$ and $A: E \rightarrow E_{1}$ and $B: E \rightarrow E_{2}$. Moreover, assume that

(A1) $A$ continuously transforms the set $T$ into $T_{1} \subset E_{1}$ and $A\left(B_{r}(E)\right)$ is bounded in $E_{1}$,

(A2c) there exists a constant $k_{1}>0$ such that $A$ satisfies the inequality

$$
c_{E_{1}}(A(U)) \leq k_{1} \cdot c_{E}(U)
$$

for arbitrary bounded subset $U$ of $T$,

(B1) $B$ continuously transforms the set $T$ into $T_{2} \subset E_{2}$ and $B\left(B_{r}(E)\right)$ is bounded in $E_{2}$,

(E1) for the triple of regular ideal spaces $\left(E, E_{1}, E_{2}\right)$ there exists a constant $k$ such that for arbitrary $x \in E_{1}$ and $y \in E_{2}$,

$$
x \cdot y \in E
$$

and

$$
\|x \cdot y\|_{E} \leq k \cdot\|x\|_{E_{1}} \cdot\|y\|_{E_{2}},
$$

(E2) $\lambda$ and $g$ are such that

$$
\|g\|_{E}+\lambda \cdot k \cdot \sup _{x \in B_{r}(E)}\|A(x)\|_{E_{1}} \cdot \sup _{x \in B_{r}(E)}\|A(x)\|_{E_{2}} \leq r
$$

for any $x \in T$ and $g+\lambda A(x) \cdot B(x) \in T$,

(C) $k \cdot k_{1} \cdot r \cdot|\lambda|<1$.

Then there exists at least one fixed point for the operator $K=g+\lambda \cdot A \cdot B$ in the set $T$ and the set of all fixed points of $K$, i.e., Fix $K$, is relatively compact in $E$. 
Proof. It is obvious that the operator $K$ is well defined on $T$ and by (E2) it takes $T$ into itself.

Denote

$$
\begin{aligned}
& M_{1}=\sup _{t \in B_{r}(E)}\|A(t)\|_{E_{1}}, \\
& M_{2}=\sup _{t \in B_{r}(E)}\|B(t)\|_{E_{2}} .
\end{aligned}
$$

Let $\left(x_{n}\right)$ be an arbitrary sequence in $T$ convergent to $x \in T$. Then

$$
\begin{aligned}
\| K\left(x_{n}\right) & -K(x) \|_{E} \\
& =|\lambda|\left\|A\left(x_{n}\right) \cdot B\left(x_{n}\right)-A(x) \cdot B(x)\right\|_{E} \\
& \leq|\lambda|\left\|A\left(x_{n}\right) B\left(x_{n}\right)-A(x) B(x)-A(x) B\left(x_{n}\right)+A(x) B\left(x_{n}\right)\right\|_{E} \\
& \leq|\lambda|\left\|\left(A\left(x_{n}\right)-A(x)\right) \cdot B\left(x_{n}\right)\right\|_{E}+|\lambda|\left\|\left(B\left(x_{n}\right)-B(x)\right) \cdot A(x)\right\|_{E} \\
& \leq k \cdot|\lambda|\left\|A\left(x_{n}\right)-A(x)\right\|_{E_{1}} \cdot M_{2}+k \cdot|\lambda|\left\|B\left(x_{n}\right)-B(x)\right\|_{E_{2}} \cdot M_{1} .
\end{aligned}
$$

From our assumptions it follows that $K$ is sequentially continuous, so it is continuous from $T$ into $E$. Now, we will investigate the contraction property for the measure $c(X)$.

Assume that $X$ is a nonempty subset of $T$ and fix an arbitrary $\varepsilon>0$. Then for an any $x \in X$ and for a set $D \subset I$ with meas $D \leq \varepsilon$, we obtain

$$
\left\|K(x) \cdot \chi_{D}\right\|_{E} \leq\left\|g \cdot \chi_{D}\right\|_{E}+k \cdot|\lambda| \cdot\left\|A(x) \cdot \chi_{D}\right\|_{E_{1}} \cdot\left\|B(x) \cdot \chi_{D}\right\|_{E_{2}} .
$$

Since $g$ has an absolutely continuous norm and since for any nonnegative realvalued functions $f$ and $g$, we have $\sup _{I}(f \cdot g) \leq \sup _{I} f \cdot \sup _{I} g$, by definition of $c(x)$ and by taking the supremum over all $x \in X$ and all measurable subsets $D$ with meas $D \leq \varepsilon$, we get

$$
\sup _{\operatorname{meas} D \leq \varepsilon} \sup _{x \in X}\left\|g(x) \cdot \chi_{D}\right\|_{E}=0
$$

and

$$
\begin{aligned}
& \sup _{\text {meas } D \leq \varepsilon} \sup _{x \in X}\left\|K(x) \cdot \chi_{D}\right\|_{E} \\
& \quad \leq k \cdot|\lambda| \cdot\left(\sup _{\text {meas } D \leq \varepsilon} \sup _{x \in X}\left\|A(x) \cdot \chi_{D}\right\|_{E_{1}} \cdot \sup _{\text {meas } D \leq \varepsilon} \sup _{x \in X}\left\|B(x) \cdot \chi_{D}\right\|_{E_{2}}\right),
\end{aligned}
$$

so

$$
c(K(X)) \leq k \cdot|\lambda| \cdot c_{E_{1}}(A(X)) \cdot c_{E_{2}}(B(X)) .
$$

As for any $x \in X$ we have

$$
\left\|x \chi_{D}\right\|_{E} \leq\|x\|_{E} \cdot\left\|\chi_{D}\right\|_{\infty}=\|x\|_{E} \leq r
$$

(cf. $\left[34\right.$, p. 66]), so $c_{E_{2}}(B(X)) \leq r$. Thus by (A2c),

$$
c(K(X)) \leq k \cdot k_{1} \cdot r \cdot|\lambda| \cdot c(X) .
$$

Recall that under our assumptions the operator $K$ maps the set $T$ which is compact in measure into itself. Because $X \subset T$ is a nonempty, bounded 
and compact in measure subset of the regular ideal space $E$, we can use Proposition 3.2 and then

$$
\beta_{H}(K(X)) \leq k \cdot k_{1} \cdot r \cdot|\lambda| \cdot \beta_{H}(X) .
$$

The inequality obtained above together with the properties of the operator $K$ and the set $T$ established before, allow us to apply the classical Darbo fixed point theorem to the measure of noncompactness $\beta_{H}$. The set of fixed points is compact; namely, if we suppose that $\beta_{H}(\operatorname{Fix} K) \neq 0$, then $K=\operatorname{Fix} K$ implies $\beta_{H}($ Fix $K)=\beta_{H}(K)<\beta_{H}(K)$, a contradiction. This completes the proof.

Note that condition (A2c) is not so restrictive as (A2) (see the comments in [10]). It is very natural and usually related to the growth condition (cf. (2.1)). Let us recall the following lemma.

Lemma 4.3 (See $\left[24\right.$, Lemma 2]). Let a continuous operator $A: L_{q}(I) \rightarrow L_{p}(I)$ satisfy the condition

$$
|A(x)(s)| \leq|b(s)|+k|x(s)|^{q / p},
$$

where $b(s) \in L_{p}(I)$ is a fixed function, and $k$ is a positive constant, for all $x$ from some ball $B_{r}\left(L_{q}(I)\right)$. Then for any subset $U \subset B_{r}\left(L_{q}(I)\right)$ the operator $A$ is a $\left.c_{L_{q}(I)}\right)_{L_{p}(I)}$ contraction on $U$ with constant $k \cdot r^{q / p}$, i.e., condition (A2c) holds true.

Remark 4.4. In contrast to the situation described above (because (A1) implies (A2c) in Lebesgue spaces), the assumption of the type (A2), i.e., contractions with respect to measures of noncompactness, is much more restrictive (it is the Lipschitz continuity of operators in Lebesgue spaces, as will be claimed below).

For the nonlinear superposition operator a very interesting discussion in this topic can be found in [3, Theorem 2]. In fact, the superposition operator $F: L_{p}(I) \rightarrow L_{p}(I)$ generated by a function $f$ satisfying the Carathéodory conditions is a contraction with respect to the (Hausdorff) measure of noncompactness if and only if it is a Lipschitz operator, i.e., $f$ is Lipschitz with respect to the second variable (for the case $p \neq q$ see [3, Theorem 3]).

An idea of comparable operators presented in [24] allows us to present an analogical result for Orlicz spaces. Again, for superposition operators condition (A2) is equivalent to the Lipschitz condition.

Lemma 4.5 (See [6, Theorem 4.10]). Let $f$ be a Carathéodory function, and suppose that the superposition operator $F$ generated by $f$ acts from $L_{\varphi}(I)$ into $L_{\varphi_{1}}(I)$. Then the Lipschitz condition for $F$ on $T$ and condition (A2) are equivalent.

Let us show some sufficient conditions for (A2c) to hold true in Orlicz spaces. 
Lemma 4.6. Suppose that $A$ is a superposition operator generated by $u$ and $A: L_{\varphi}(I) \rightarrow E_{\varphi_{1}}(I)$ is continuous. Then the condition

$$
|u(t, x)| \leq b(t)+d \varphi_{1}^{-1}\left[\varphi\left(\frac{x}{r}\right)\right], \quad t \in I, x \in \mathbb{R},
$$

where $b \in E_{\varphi_{1}}(I)$ and $r, d \geq 0$, implies that $A$ is a $c_{L_{\varphi}(I)^{-}} c_{L_{\varphi_{1}}(I)}$ contraction with constant $k_{1}=d / r$. Thus (A2c) holds true on $B_{r}\left(L_{\varphi}(I)\right)$.

Proof. Define a new operator

$$
A_{0}(x)=b+d \cdot \varphi_{1}^{-1}\left[\varphi\left(\frac{x}{r}\right)\right] .
$$

Clearly it acts from $L_{\varphi}(I)$ into $E_{\varphi_{1}}(I)$ too. The two operators are comparable in the sense of [24], so

$$
c_{L_{\varphi}(I)}(A(U)) \leq c_{L_{\varphi}(I)}\left(A_{0}(U)\right) .
$$

But for any $\varepsilon>0$,

$$
\left\|A_{0}(x) \chi_{D}\right\|_{\varphi_{1}} \leq\left\|b \chi_{D}\right\|_{\varphi_{1}}+d\left\|\varphi_{1}^{-1}\left(\varphi\left(\frac{x}{r}\right)\right) \chi_{D}\right\|_{\varphi_{1}}
$$

for any measurable subset $D$ of $I$ with meas $D \leq \varepsilon$ and $x \in B_{r}\left(L_{\varphi}(I)\right)$.

Recall that for $x \in B_{r}\left(E_{\varphi}(I)\right)$ we have $x / r \in B_{1}\left(E_{\varphi}(I)\right)$ so

$$
\int_{I} \varphi\left(\frac{x(s)}{r}\right) d s \leq\left\|\frac{x}{r}\right\|_{\varphi}=\frac{1}{r}\|x\|_{\varphi} .
$$

As

$$
\int_{I} \varphi_{1}\left(\varphi_{1}^{-1}\left[\varphi\left(\frac{x(s)}{r}\right)\right]\right) d s=\int_{I} \varphi\left(\frac{x(s)}{r}\right) d s
$$

and since we have ideal spaces, for any measurable subset $D \subset I$, we get

$$
\begin{aligned}
\left\|A_{0}(x) \chi_{D}\right\|_{\varphi_{1}} & \leq\left\|b \chi_{D}\right\|_{\varphi_{1}}+d\left\|\varphi_{1}^{-1}\left[\varphi\left(\frac{x}{r}\right)\right] \chi_{D}\right\|_{\varphi_{1}} \\
& \leq\left\|b \chi_{D}\right\|_{\varphi_{1}}+d \frac{1}{r}\left\|x \chi_{D}\right\|_{\varphi} .
\end{aligned}
$$

Let $U \subset B_{r}\left(E_{\varphi}(I)\right)$. By taking the supremum over all $x \in U$ and all measurable subsets $D$ with meas $D \leq \varepsilon$, as $b \in E_{\varphi_{1}}(I)$, we obtain

$$
c_{L_{\varphi_{1}}}(A(U)) \leq c_{L_{\varphi_{1}}}\left(A_{0}(U)\right) \leq d \frac{1}{r} c_{L_{\varphi}}(U)
$$

Remark 4.7. We need to remark that (A2c) can still be easily reformulated. Denote by $E_{0}$ a regular part of $E$ (a closure of $L^{\infty}(I)$ in $E$ ). It is sufficient to assume that $K: T \cap E_{0} \rightarrow T \cap E_{0}$. This seems to be important for the case of the so-called improving operators (taking bounded subsets of $E$ into the sets with equiabsolutely continuous norms, i.e., into $\left.E_{0}\right)$. A detailed theory of compactness in regular ideal spaces can be found in [33].

If the operator takes bounded subsets of $E$ into the family of sets with equiabsolutely continuous norms in $E_{1}$, it will be called generalized improving.

Corollary 4.8. If the operator $A$ is generalized improving, then (A2c) holds true with $k_{1}=0$. 
It was observed that a compactness criterion for Lebesgue spaces requires some uniform integrability condition, as well as a condition of compactness in measure (see [22] or [26] for the discussion). Thus, the next goal of the paper is to indicate a big class of sets which are compact in measure.

Remark 4.9. There are many natural sets which are compact in measure. Let us observe that at least a specific class of sets should be interesting for integral (and differential equations), namely sets consisting of a.e. monotonic functions. This observation is well known (cf. [8] or [27]). Surprisingly, to the best of our knowledge, there is no published proof, even for sets of continuous functions.

We present this proof in a general regular ideal space. It is also a general scheme showing how to construct more sets that are compact in measure. Note that this is a weak version of Helly's selection theorem (cf. $[8,16])$.

Lemma 4.10. Assume that a bounded set $U$ is a subset of a regular ideal space $E$ of real-valued functions over a bounded interval $I$ such that all the functions from $U$ are a.e. monotonic. Then this set is compact in measure in the space $E$.

Proof. Let us recall that a topology of convergence in measure is a metric topology (cf. [25, 26]), so we only need to check sequential compactness. Without loss of generality, let us restrict to the case of a.e. increasing functions. Take an arbitrary sequence $\left(x_{n}\right) \subset U$. Denote by $W$ the set such that $x_{n}$ are increasing on $J=I \backslash W$. Let $Z$ be a dense subset of $J$. Since $\left\{x_{n}(t)\right\}$ is bounded on $Z$ (except perhaps the point $t=b$ ), by a diagonal procedure we can subtract a pointwise convergent subsequence $\left(x_{n_{k}}\right)$ of $\left(x_{n}\right)$ (on $Z$ ). Then the limit $x=\lim _{k \rightarrow \infty} x_{n_{k}}$ is an increasing function on $Z$. It is known that this function can be extended to $y$ defined on $J$ in such a way $y$ is a limit of $\left(x_{n_{k}}\right)$ on $Z$.

Let $t_{0}$ be an arbitrary point in $J$. Since $Z$ is dense in $J$, we are able to find two sequences $\left(s_{n}\right)$ and $\left(\tau_{n}\right)$ of points in $Z$ tending to $t_{0}$ such that $s_{n}<t_{0}<\tau_{n}$. For any fixed $k \in \mathbb{N},\left(x_{n_{k}}\right)$ is increasing on $J$ and we have

$$
x_{n_{k}}\left(s_{n}\right)<x_{n_{k}}\left(t_{0}\right)<x_{n_{k}}\left(\tau_{n}\right),
$$

and passing to the limit with $k \rightarrow \infty$ we obtain

$$
y\left(s_{n}\right) \leq \liminf _{k \rightarrow \infty} x_{n_{k}}\left(t_{0}\right) \leq \limsup _{k \rightarrow \infty} x_{n_{k}}\left(t_{0}\right) \leq y\left(\tau_{n}\right) .
$$

By passing to the limit with $n \rightarrow \infty$ we get

$$
y\left(t_{0}-\right) \leq \liminf _{k \rightarrow \infty} x_{n_{k}}\left(t_{0}\right) \leq \limsup _{k \rightarrow \infty} x_{n_{k}}\left(t_{0}\right) \leq y\left(t_{0}+\right) .
$$

For any point of continuity of $y$ we have $y(t)=\lim _{k \rightarrow \infty} x_{n_{k}}(t)$. The set $D$ of all points of discontinuity of this function is at most countable. Then $y$ is a.e. increasing. 
Since the measure of $I$ is finite, the sequence $\left(x_{n}\right)$ contains an a.e. convergent subsequence which is also convergent in measure (by the Lebesgue theorem). Finally, an arbitrary sequence in $U$ contains a subsequence convergent in measure to some $y \in U$ and then this set is compact in measure.

It is clear that the subfamily of $U$ consisting of positive functions is compact in measure too (it is an important property when studying positive monotonic solutions for some problems). In such a case we can apply our theorem to the sets $U$ consisting of positive functions.

Corollary 4.11. Take an arbitrary closed convex cone $K$ in $E$. Then $U \cap K$ is also compact in measure. In particular, for a cone

$$
K_{0}=\{x \in E: x \geq 0 \text { a.e. on } I\}
$$

the set $U \cap K_{0}$ is compact in measure.

We have to illustrate our approach, so our example of applications need not be presented for very general and complicated problems. Since the technical details can obscure the main ideas, we concentrate here on the acting and continuity conditions for operators in some ideal spaces.

Let us recall some problems mentioned in the Introduction for which our approach allows to find discontinuous solutions. Let us consider a class of problems motivated by the Chandrasekhar equation

$$
x(t)=1+x(t) \int_{0}^{1} \frac{t}{t+s} \psi(s) x(s) d s,
$$

which is one of the prototypes for our study.

In order to solve the above equation by applying earlier results, it is necessary to impose the condition that the so-called characteristic function $\psi$ is a polynomial (as in the book by Chandrasekhar [15, Chapter 5]) or at least continuous (cf. [14, Theorem 3.2], [7]). This function is immediately related to the angular pattern for single scattering, and then our results allow to consider some peculiar states of the atmosphere. In astrophysical applications of the Chandrasekhar equation the only restriction that $\int_{0}^{1} \psi(s) d s \leq 1 / 2$ is treated as necessary (cf. [14, Corollary 2, Chapter VIII, p. 187]). The continuity assumption for $\psi$ implies the continuity of solutions for the considered equation (cf. [14]) and then it seems to be too restrictive even from the theoretical point of view.

Let us try to prove the existence of solutions of equation (1.1) which is general than the Chandrasekhar one. Since for this equation we should expect positive solutions, we will prove the existence of such solutions for (1.1). Thus we are interested in solutions that are simultaneously discontinuous and positive. If possible, we will try to find solutions more regular than integrable, namely in Orlicz spaces. In comparison to earlier results (cf. [18]) we simplify the proof, by dividing it into parts related to the assumptions of Theorem 4.2. Moreover, we are able to obtain some extension by proving the existence of positive solutions and by relaxing some assumptions. 
In the considered problem we have a superposition operator

$$
A(x)(t)=u(t, x(t))
$$

and an integral operator

$$
B x(t)=\int_{0}^{1} k(t, s) f(s, x(s)) d s .
$$

Let us start by presenting some assumptions allowing us to apply Theorem 4.2, as well as some comments about it.

Take a triple of Orlicz spaces $\left(L_{\varphi}(I), L_{\varphi_{1}}(I), L_{\varphi_{2}}(I)\right)$ satisfying (E1) (see Lemmas 2.1 and 2.2). Recall that for a given $L_{\varphi}(I)$, it is not uniquely determined and can be chosen in accordance with Lemmas 2.1 and 2.2. It should be related to the growth conditions for the considered operators (in such a case $\varphi_{1}$ and $\varphi_{2}$ are growing more rapidly than $\varphi$ - cf. [28, Theorem 13.6]). We need to assume that $A$ is a superposition operator from Orlicz spaces $L_{\varphi}(I)$ into $L_{\varphi_{1}}(I)$. Recall that any Orlicz space $L_{\varphi}(I)$ is ideal and if $\varphi$ satisfies the $\Delta_{2}$-condition, it is also regular (cf. [4, Theorem 1]). Note that in such a case the superposition operator $A$ acting from $L_{\varphi}(I)$ into $L_{\varphi_{1}}(I)$ is bounded on balls and, moreover, we have the following lemma.

Lemma 4.12 (See [28, Theorem 17.6]). Suppose that the function $u: I \times \mathbb{R} \rightarrow$ $\mathbb{R}$ satisfies the Carathéodory conditions and

$$
|u(t, x)| \leq b(t)+d \varphi_{1}^{-1}\left[\varphi\left(\frac{x}{r}\right)\right], \quad t \in I, x \in \mathbb{R},
$$

where $b \in E_{\varphi_{1}}$ and $r, d \geq 0$. If the $N$-function $\varphi_{1}$ satisfies the $\Delta_{2}$-condition, then the superposition operator $A(x)(\cdot)=u(\cdot, x(\cdot))$ generated by $u$ acts from $B_{r}\left(E_{\varphi}(I)\right)$ into the space $L_{\varphi_{1}}(I)=E_{\varphi_{1}}(I)$ and is continuous.

As the space $E_{\varphi}(I)$ is a regular part of the Orlicz space $L_{\varphi}(I)$ (cf. [34, p. 72]), in the context of Orlicz spaces, we have a result about the continuity of $A$ (assumption (A1)).

Lemma 4.13 (See [19]). Let u be a Carathéodory function. If the superposition operator $A$ acts from $L_{\varphi}(I)$ into $E_{\varphi_{1}}(I)$, then it is continuous.

If we keep the hypothesis of Lemma 4.12, then Lemma 4.6 implies that the operator $A$ is bounded on balls with small radius, i.e.,

$$
\sup _{x \in B_{r}\left(L_{\varphi}(I)\right)}\|A(x)\|_{L_{\varphi_{1}}(I)}
$$

is finite and then condition (A2c) holds true.

To check condition (E2) we can apply Lemma 4.6, so condition (4.2) will allow us to check condition (E2), it holds true for $k_{1}=k \cdot(1 / r)$.

One more comment about assumption (E2) in Theorem 4.2: Since the characteristic function is integrable in any Orlicz space, we can treat $g$ as $\chi_{I}$, so $\|g\|_{\varphi}=\operatorname{meas}(I) \cdot \psi^{-1}(1 / \operatorname{meas}(I))$ (the Orlicz norm), when $\psi$ is a complementary function to $\varphi$ (or $1 / \varphi^{-1}(1 / \operatorname{meas}(I))$ for the Luxemburg norm), so it need not be equal one and (E2) can be fulfilled for the considered equation (see [28, Section 9.3]). Note that any Orlicz space can be renormed in such 
a way to use the most convenient norm from the class of equivalent norms (cf. [28, Section 13.2]).

For the operator $B$ we still need to assume (B1). Let us recall that a linear operator $B_{0}$ with kernel $k(t, s)$ maps some Orlicz space $L_{\Phi}(I)$ into $L_{\varphi_{2}}(I)$ and it is continuous if the kernel belongs to a specific Orlicz space, say $L_{\Psi}$ (for a detailed study see [28, Theorems 15.1, 15.4 and Lemmas 15.1, 15.2]). Relative to the case of the Chandrasekhar equation (4.1) we do not need to assume that $\psi$ in (4.1) is continuous.

Assume that $f$ generates the superposition operator $F$ acting from $L_{\varphi}(I)$ into $L_{\Psi}(I)=E_{\Psi}(I)$, where $\Phi$ and $\Psi$ are complementary $N$-functions. Whenever $\Psi$ satisfies the $\Delta_{2}$-condition, $L_{\Psi}(I)$ is regular and consequently it is continuous and $F$ is bounded on $B_{r}\left(L_{\varphi}(I)\right)$. Thus $F$ continuously maps $L_{\varphi}(I)$ into $L_{\Psi}(I)$. In particular, it can be done when $L_{\Psi}(I)$ and $L_{\Phi}(I)$ are Lebesgue spaces $L^{p}(I)$ and $L^{q}(I)$, respectively, satisfying $1 / p+1 / q=1$.

If $B_{0}$ continuously maps $L_{\Phi}(I)$ into $L_{\varphi_{2}}(I)$, then for $B=B_{0} \circ F$ we have that $B: L_{\varphi}(I) \rightarrow L_{\varphi_{2}}(I)$ is continuous and bounded on a ball $B_{r}\left(L_{\varphi}(I)\right)$. The fundamental theorem of continuity for linear operators $B_{0}$ is presented in [28, Theorem 15.4]. It is worth noting that the conditions presented in this theorem are not equivalent, so it is impossible to put a single condition on the kernel $k$ allowing to prove its thesis. To keep the general idea of the paper, we use this assumption as is. In such a situation the operator $B$ is an operator from $L_{\varphi}(I)$ into $L_{\varphi_{2}}(I)$, continuous and bounded on a ball $B_{r}\left(L_{\varphi}(I)\right)$. An appropriate choice of $r$ allows us to take this ball invariant under $B$. In such a case (B1) is satisfied.

To ensure that our operators transform our set $T$ (consisting of a.e. nondecreasing positive functions) from the ball $B_{r}\left(L_{\varphi}(I)\right)$ into itself, we need to have monotonicity preserving operators.

For the integral operator of the form $B_{0}(x)(t)=\int_{I} k(t, s) x(s) d s$ we have the following theorem due to Krzyż [29].

Lemma 4.14 (See [29, Theorem 6.2]). The operator $B_{0}$ preserves the monotonicity of functions if and only if

$$
\int_{0}^{b} k\left(t_{1}, s\right) d s \geq \int_{0}^{b} k\left(t_{2}, s\right) d s
$$

for $t_{1}<t_{2}, t_{1}, t_{2} \in I$ and for any $b \in I$.

Let us remark that to apply our main theorem we need to have

$$
c_{L_{\varphi_{1}}(I)}(A(X))=\beta_{H}(A(X)),
$$

where the set $A(X)$ should be compact in measure. Recall that the superposition operator generated by a function satisfying the Carathédory conditions and acting between Orlicz spaces maps sequences of functions convergent in measure into sequences convergent in measure (cf. [31]). We will use the following lemma (cf. [18, Proposition 4.1]).

Lemma 4.15. Assume the function $u: I \times \mathbb{R} \rightarrow \mathbb{R}$ satisfies the Carathéodory conditions, the function $t \rightarrow u(t, x)$ is a.e. nondecreasing on a finite interval $I$ 
for each $x \in \mathbb{R}$ and the function $x \rightarrow u(t, x)$ is a.e. nondecreasing on $\mathbb{R}$ for any $t \in I$. Assume that $A: L_{\varphi}(I) \rightarrow E_{\varphi_{1}}(I)$. Then $A(X)$ is compact in measure for an arbitrary bounded and compact in measure subset $X$ of $L_{\varphi}(I)$.

Finally, we need to take a positive function $g \in E_{\varphi}(I)$ and both $A$ and $B$ should be positive (i.e., positivity preserving operators, see [27], for instance). In particular, an operator $B$ from the Chandrasekhar equation (4.1) is positive whenever $\psi$ is a positive function.

Let us observe that the appropriate choice of $r>0$ allows us to fulfill assumption (C) for sufficiently small $\lambda$.

By gathering all the assumptions considered above we have an immediate consequence of Theorem 4.2.

Theorem 4.16. Assume that $\varphi, \varphi_{1}, \varphi_{2}$ are $N$-functions and $g \in E_{\varphi}(I)$ is an a.e. nondecreasing positive function. The $N$-functions $\Psi$ and $\Phi$ are complementary, with $\Psi$ satisfying the $\Delta_{2}$-condition. Moreover, assume that

(C1) there exists a constant $k>0$ such that for every $u \in L_{\varphi_{1}}(I)$ and $w \in L_{\varphi_{2}}(I)$ we have $\|u w\|_{\varphi} \leq k\|u\|_{\varphi_{1}}\|w\|_{\varphi_{2}}$, where the $N$-function $\varphi_{1}$ satisfies the $\Delta_{2}$-condition,

(C2) the function $u: I \times \mathbb{R} \rightarrow \mathbb{R}_{+}$satisfies the Carathéodory conditions and $u(t, x)$ is nondecreasing with respect to both variables $t$ and $x$ separately,

(C3) the function $u$ satisfies the following growth condition:

$$
|u(t, x)| \leq b(t)+d \varphi_{1}^{-1}\left[\varphi\left(\frac{x}{r}\right)\right], \quad t \in I, x \in \mathbb{R},
$$

where $b \in E_{\varphi_{1}}(I)$ for some $r, d \geq 0$,

(C4) $s \rightarrow k(t, s) \in L_{\Phi}(I)$ for a.e. $t \in I$ and $p(t)=\|k(t, \cdot)\|_{\Phi} \in E_{\varphi_{2}}(J)$.

Moreover,

$$
\int_{0}^{b} k\left(t_{1}, s\right) d s \geq \int_{0}^{b} k\left(t_{2}, s\right) d s
$$

for $t_{1}<t_{2}, t_{1}, t_{2} \in I$ and for any $b \in I$,

(C5) the function $f: I \times \mathbb{R} \rightarrow \mathbb{R}_{+}$satisfies the Carathéodory conditions and $f(t, x)$ is nondecreasing with respect to both variables $t$ and $x$ separately. Moreover, a superposition operator $F$ generated by $f$ continuously maps $L_{\varphi}(I)$ into the Orlicz space $E_{\Psi}(I)$,

(C6) the constant $\lambda$ satisfies the inequality

$$
|\lambda|<\frac{1}{k \cdot d}
$$

and $g$ is such that

$$
\begin{aligned}
\|g\|_{L_{\varphi_{1}(I)}}+\lambda \cdot k & \cdot \sup _{x \in B_{r}\left(L_{\varphi}(I)\right)}\|A(x)\|_{L_{\varphi_{1}}(I)} \\
& \cdot \sup _{x \in B_{r}\left(L_{\varphi(I)}\right)}\|B(x)\|_{L_{\varphi_{2}}(I)} \leq r .
\end{aligned}
$$

Then there exists an a.e. nondecreasing positive solution $x \in E_{\varphi}(I)$ of (1.1) on the interval $I$. 
In particular, besides the results for continuous solutions, the above result is an extension of [19, Corollary 5.2] (from $L_{p}$-solutions to positive $L_{\varphi}$-solutions) and to some extent [19, Theorem 5.1]. For the nonquadratic integral equations with positive solutions in Orlicz spaces we refer the reader to $[1$, Theorem 4.2] (see also [2, Chapter 17]).

Another typical example of quadratic operator equation considered in earlier papers is the Cauchy problem:

$$
\left(\frac{x(t)-g(t)}{f_{1}(t, x)}\right)^{\prime}=f_{2}(t, x(t)), \quad x(0)=0,
$$

for $f_{1}: I \times \mathbb{R} \rightarrow \mathbb{R} \backslash\{0\}$ and where $f_{2}$ and $g$ satisfy some regularity conditions, considered for instance in [21]. This problem can be rewritten in a form of quadratic integral equations (see [21]), so our theorems apply. When we are looking for continuous solutions for quadratic integral equations, the solutions for the above differential problems are classical. We stress that our approach can be used to investigate weaker types of solutions (in Orlicz-Sobolev spaces, for instance).

\section{References}

[1] R. Agarwal, D. O'Regan and P. Wong, Constant-sign solutions of a system of Volterra integral equations in Orlicz spaces. J. Integral Equations Appl. 20 (2008), 337-378.

[2] R. Agarwal, D. O'Regan and P. Wong, Constant-Sign Solutions of Systems of Integral Equations. Springer, New York, 2013.

[3] J. Appell, Implicit functions, nonlinear integral equations, and the measure of noncompactness of the superposition operator. J. Math. Anal. Appl. 83 (1981), $251-263$.

[4] J. Appell, The importance of being Orlicz. Banach Center Publ. 64 (2004), $21-28$.

[5] J. Appell and E. De Pascale, Su alcuni parametri connessi con la misura di non compatteza di Hausdorff in spazi di funzioni misurabili. Boll. Unione Mat. Ital. B 3 (1984), 497-515.

[6] J. Appell and P. P. Zabreiko, Nonlinear Superposition Operators. Cambridge University Press, Cambridge, 1990.

[7] I. K. Argyros, On a class of quadratic integral equations with perturbation. Funct. Approx. Comment. Math. 20 (1992), 51-63.

[8] J. Banaś, Integrable solutions of Hammerstein and Urysohn integral equations. J. Aust. Math. Soc. Ser. A 46 (1989), 61-68.

[9] J. Banaś, Applications of measures of weak noncompactness and some classes of operators in the theory of functional equations in the Lebesgue space. Nonlinear Anal. 30 (1997), 3283-3293.

[10] J. Banaś and W. G. El-Sayed, Measures of noncompactness and solvability of an integral equation in the class of functions of locally bounded variation. J. Math. Anal. Appl. 167 (1992), 133-151. 
[11] J. Banaś and K. Goebel, Measures of Noncompactness in Banach Spaces. Lect. Notes Math. 60, Marcel Dekker, New York, 1980.

[12] J. Banaś and M. Lecko, Fixed points of the product of operators in Banach algebra. Panamer. Math. J. 12 (2002), 101-109.

[13] J. Banaś and K. Sadarangani, Solutions of some functional-integral equations in Banach algebras. Math. Comput. Modelling 38 (2003), 245-250.

[14] J. Caballero, A. B. Mingarelli and K. Sadarangani, Existence of solutions of an integral equation of Chandrasekhar type in the theory of radiative transfer. Electron. J. Differential Equations 57 (2006), 1-11.

[15] S. Chandrasekhar, Radiative Transfer. Dover Publications, New York, 1960.

[16] V. V. Chistyakov, A Banach algebra of functions of several variables of finite total variation and Lipschitzian superposition operators. I. Nonlinear Anal. 62 (2005), 559-578.

[17] M. Cichoń and M. Metwali, On quadratic integral equations in Orlicz spaces. J. Math. Anal. Appl. 387 (2012), 419-432.

[18] M. Cichoń and M. Metwali, On monotonic integrable solutions for quadratic functional integral equations. Mediterr. J. Math. 10 (2013), 909-926.

[19] M. Cichoń and M. Metwali, Existence of monotonic $L_{\varphi}$-solutions for quadratic Volterra functional-integral equations. Electron. J. Qual. Theory Differ. Equ. (2015), No. 13, 1-16.

[20] B. C. Dhage, Remarks on two fixed-point theorems involving the sum and the product of two operators. Comput. Math. Appl. 46 (2003), 1779-1785.

[21] B. C. Dhage and M. Kumpulainen, Nonlinear functional boundary value problems involving the product of two nonlinearities. Appl. Math. Lett. 21 (2008), $537-544$.

[22] N. Erzakova, On measures of non-compactness in regular spaces. Z. Anal. Anwend. 15 (1996), 299-307.

[23] N. Erzakova, Compactness in measure and measure of noncompactness. Sib. Math. J. 38 (1997), 926-928.

[24] N. Erzakova, On measure-compact operators. Russian Math. 55 (2011), 37-42.

[25] L. C. Florescu, The centennial of convergence in measure. Sci. Stud. Res. Ser. Math. Inform. 19 (2009), 221-239.

[26] L. C. Florescu and Ch. Godet-Thobie, Young Measures and Compactness in Measure Spaces. Walter de Gruyter, Berlin, 2012.

[27] M. A. Krasnosel'skii, P. P. Zabreiko, J. I. Pustyl'nik and P. E. Sobolevskii, Integral Operators in Spaces of Summable Functions. Noordhoff, Leyden, 1976.

[28] M. A. Krasnosel'skii and Yu. Rutitskii, Convex Functions and Orlicz Spaces. Noordhoff, Groningen, 1961.

[29] J. Krzyż, On monotonicity-preserving transformations. Ann. Univ. Mariae Curie-Skłodowska Sect. A 6 (1952), 91-111.

[30] L. Maligranda, Orlicz Spaces and Interpolation. Departamento de Matemática, Universidade Estadual de Campinas, Campinas, 1989.

[31] R. Płuciennik, The superposition operator in Musielak-Orlicz spaces of vectorvalued functions. In: Proceedings of the 14th Winter School on Abstract Analysis, Circolo Matematico di Palermo, 1987, 411-417.

[32] M. Väth, Ideal Spaces. Lect. Notes Math. 1664, Springer, Berlin, 1997. 
[33] M. Väth, A Compactness criterion of mixed Krasnoselskii-Riesz type in regular ideal spaces of vector functions. Z. Anal. Anwend. 18 (1999), 713-732.

[34] M. Väth, Volterra and Integral Equations of Vector Functions. Marcel Dekker, New York, 2000.

Mieczysław Cichoń

Faculty of Mathematics and Computer Science

A. Mickiewicz University

Umultowska 87

61-614 Poznań

Poland

e-mail: mcichon@amu.edu.pl

Mohamed M. A. Metwali

Department of Mathematics

Faculty of Sciences

Damanhour University

Egypt

e-mail: m.metwali@yahoo.com

Open Access This article is distributed under the terms of the Creative Commons Attribution 4.0 International License (http://creativecommons.org/licenses/by/4.0/), which permits unrestricted use, distribution, and reproduction in any medium, provided you give appropriate credit to the original author(s) and the source, provide a link to the Creative Commons license, and indicate if changes were made. 